\section{POS0379 DETERMINING IN WHICH PRECLINICAL STAGE HLA-SHARED EPITOPE ALLELES AND SMOKING EXERT THEIR EFFECT IN THE DEVELOPMENT OF RHEUMATOID ARTHRITIS}

F. Wouters ${ }^{1}$, M. Maurits ${ }^{2}$, L. van Boheemen ${ }^{3}$, M. Verstappen ${ }^{2}$, X. Matthijssen ${ }^{2}$, E. Niemantsverdriet ${ }^{2}$, R. Knevel ${ }^{2}$, D. van Schaardenburg ${ }^{3}$, R. Toes $^{2}$, A. van der Helm - van Mili ${ }^{2,4} .{ }^{1}$ Leiden University Medical Centre, Department of Rheumatology, Leiden, Netherlands; ${ }^{1}$ Leiden University Medical Centre, Department of Rheumatology, Leiden, Netherlands; ${ }^{3}$ Amsterdam Rheumatology and Immunology Centre, Reade, Department of Rheumatology, Amsterdam, Netherlands; ${ }^{4}$ Erasmus University Medical Centre, Department of Rheumatology, Rotterdam, Netherlands

Background: The HLA shared epitope (SE) and smoking are the best known genetic and environmental risk factors for rheumatoid arthritis (RA) development; however, at which pre-RA stage they exert their effect is unknown. The following stages are discerned: an asymptomatic stage in which autoimmune responses can develop, a symptomatic stage (clinically suspect arthralgia (CSA)), and development of clinically apparent inflammatory arthritis (IA). Studies in the general asymptomatic population revealed contrasting results on the associations between SE-alleles and smoking and the presence of anti-citrullinated protein antibodies (ACPA). Furthermore, studies on these risk factors in the symptomatic pre-RA phase are scarce and these data might teach us whether SE-alleles and smoking are involved in symptom development and/or progression to clinical arthritis.

Objectives: We aimed to determine at which pre-RA stage SE and smoking exert their effect. In this respect, the analyses were focused on the presence of ACPA, but associations for other anti-modified protein antibodies (anti-carbamylated and anti-acetylated protein antibodies (anti-CarP and AAPA, respectively)) were also studied.

Methods: Results from the literature on the association of SE and smoking with ACPA in the asymptomatic population were summarized in inverse-variance weighted meta-analyses. In addition 577 CSA-patients were studied. Associations of SE and smoking with IgG ACPA were studied at baseline (CSA-onset), to assess an effect on symptom development. Additionally, patients were monitored for the development of clinically apparent inflammatory arthritis (IA) for median 2 years and associations of SE, smoking and auto-antibodies with progression to IA were determined. Analyses were stratified for ACPA-status and associations in ACPA-positive patients were validated in meta-analyses with other arthralgia-cohorts. Finally analyses were repeated for anti-CarP and AAPA.

Results: Meta-analyses showed that SE is not associated with ACPA-positivity in the asymptomatic population (OR $1.06(95 \% \mathrm{Cl} 0.69-1.64))$, whereas smoking was associated (OR 1.37 (1.15-1.63)). At CSA-onset, both SE and smoking associated with ACPA-positivity (OR 2.08 (1.24-3.49) and OR 2.41 (1.31-4.43), respectively). During follow-up of CSA-patients SE associated with IA-development (HR 1.86 (1.23-2.82)), in contrast to smoking. SE conferred risk for IA-development in ACPA-negative CSA-patients (HR 1.71 (0.99-2.96)) and in ACPA-positive patients (CSA-cohort HR 1.29 (0.67-2.47); meta-analysis three arthralgia-cohorts HR 1.52 (1.08-2.15)). Investigating the other autoantibodies revealed that SE and smoking were not associated with anti-CarP or AAPA-positivity at CSA-onset; longitudinally AAPA associated with progression to IA independent from ACPA and RF (HR 1.79 (1.02-3.16)), whilst anti-Carp did not.

Conclusion: SE and smoking act in partly different pre-RA stages. Although SE does not associate with ACPA in the general population, it does mediate symptom-development and further progression to clinical arthritis. Smoking confers risk to development of ACPA and/or joint symptoms, but is not further involved in IA-development. The time-specific biologic pathways that are underlying need further exploration. These data enhance the understanding of the timing of key genetic and environmental risk factors in the trajectory of RA development. Disclosure of Interests: None declared

DOI: 10.1136/annrheumdis-2021-eular.1342

\section{POS0380 HORMONES OF ADIPOSE TISSUE IN PATIENTS WITH RHEUMATOID ARTHRITIS WHO HAVE REDUCED BODY WEIGHT}

L. Arefeva ${ }^{1}$, G. Kravtsov ${ }^{2}$, V. Polyakov ${ }^{2}$, V. Kravtsov3, L. Seewordova ${ }^{4}$. ${ }^{1}$ Volgograd State Medical University, Cardiology of University Clinical, Volgograd, Russian Federation; ${ }^{2}$ Volgograd State Medical University, University Clinical, Volgograd, Russian Federation; ${ }^{1}$ Volgograd State Medical University, Cardiology of University Clinical, Volgograd, Russian Federation; ${ }^{4}$ Federal State Budgetary Institution "Zborovsky Research Institute of Clinical and Experimental Rheumatology", Laboratory Methods of Treatment and Prevention of Joint Diseases, Volgograd, Russian Federation

Background: Overweight in patients with rheumatic diseases is a condition that prolongs chronic inflammation and promotes synthesis and secretion of pro-inflammatory factors by adipose tissue, such as classical cytokines, tumor necrosis factor- $\alpha$ (TNF- $\alpha$ ), adipokines (leptin, adiponektin, resistin) and other newly identified proinflammatory factors (fetuin A, nesfatin, hemerin, lipokain, serum amyloid protein 3) $[1,2,3,4]$.

Objectives: We investigated the relationship the effect of weight loss over $5 \mathrm{~kg}$ on the clinical manifestations of arthritis and hormones of adipose tissue serum levels in patients with rheumatoid arthritis (RA).

Methods: We observed 80 female patients with RA (EULAR/ARA 2010 criteria) ranged in age from 39 to 69 years (mean age $51,72 \pm 5,83$ years) and the contro group (60 healthy persons) with no complaints of pain in the joints over a lifetime, and without clinical signs of RA. Fetuin A, nesfatin, hemerin, leptin, adiponektin, resistin, visfatin level in serum was determined by ELISA-test using a commercial test systems.

Results: As overweight patients were recruited in the study, hypocaloric diet low in animal fats and physiotherapy has been recommended to all participants. The positive dynamics in body weight loss over $5 \mathrm{~kg}$ within 3 months has been achieved by 34 patients $(27,2 \%)$. In RA patients with weight loss, a significant decrease in the serum level of pro-inflammatory cytokines (fetuin $A$, nesfatin, hemerin, leptin, adiponektin, resistin, visfatin $(p<0.01))$ and an increase in the quality of life according to the EQ-5D-5L $(p<0.001)$ index were observed. This fact is probably explained by the decreased activity of inflammatory process after RA therapy and weight reduction.

Conclusion: Thus, as a result of our study patients with RA with weight loss of more than $5 \mathrm{~kg}$ had more obvious pain relief than patients with the original weight. These findings suggest that there is a possible role of tissue pro-inflammatory cytokines in the pathogenesis of rheumatoid arthritis. All patients with RA with a BMl over $25 \mathrm{~kg} / \mathrm{m}^{2}$ are recommended to lower their weight to decrease the mechanical stress on the joints, and also to reduce the severity of inflammation and metabolic disorders.

\section{REFERENCES:}

[1] Akhverdyan, Y. et al. The nicotinamide-phosphoribosiltransferase as a marker of systemic inflammation under osteoarthrosis // Klin Lab Diagn. 2017; 62(10):606-610.

[2] Kravtcov, V. et al. High level of adipokines and overweight as factors contributing to osteoarthritis progression // Osteoporosis International, 2019. V.30 (2). S. 408.

[3] Papichev, E. V. et al. Parameters of mineral-bone metabolism and fetuin-A level in patients with rheumatoid arthritis // Osteoporosis International, 2019. V.30 (2), S.381

[4] Polyakova J. et al. Tissue cytokines and their role in the pathogenesis of rheumatic diseases // Annal.Rheum.Diseases, 2019. T. 30 (2), № S.387.

Disclosure of Interests: None declared

DOI: 10.1136/annrheumdis-2021-eular.1432

\section{POS0381 PATIENTS AT RISK FOR RA SHOW THE SAME AMOUNT OF ACUTE SOLUBLE CARTILAGE DEGRADATION MARKERS AFTER PHYSICAL ACTIVITY COMPARED TO PATIENTS WITH ESTABLISHED RA}

L. Bleckwedel-Rolack ${ }^{1}$, K. Tascilar ${ }^{1}$, V. Nees ${ }^{1}$, J. Hühne ${ }^{1}$, A. Hueber ${ }^{2}$, J. Rech ${ }^{1}$, G. Schett ${ }^{1}$, A. Kleyer ${ }^{1}$, A. M. Liphardt ${ }^{1} .{ }^{1}$ Friedrich-Alexander-University Erlangen-Nürnberg \& University Hospital Erlangen, Internal Medicine 3 Rheumatology \& Immunology, Erlangen, Germany; ${ }^{2}$ Sozialstiftung Bamberg - Klinikum am Bruderwald, Rheumatology, Bamberg, Germany

Background: Serum concentration of cartilage oligomeric matrix protein (COMP) is related to the degree of cartilage destruction in patients with rheumatoid arthritis (RA) and shows a mechanosensitive response to ambulatory loads. We showed previously, that individuals with positive for anti-citrullinated protein antibody (ACPA+) status already show bone loss. It is unclear if these individuals experience cartilage deterioration and how this is related to physical activity.

Objectives: To test whether soluble COMP levels in ACPA+ display the same response as RA patients after a walking exercise and explore the association between overall serum COMP levels and physical activity.

Methods: RA patients and ACPA+ individuals (IRACE cohort: Individuals at Risk for Arthritis Cohort Erlangen; Ethics approval 334_16B) were enrolled in the study after written informed consent. Inclusion criteria were age between 18 and 69 years, RA (by 2010 ACR/EULAR criteria) or ACPA+ (without clinical manifestation of RA and prior treatment with glucocorticoids, DMARDs and biologics). The study comprised three visits (baseline (Visit 1), 6 (Visit 2), and 12 months (Visit 3). During each visit, serum samples were collected after 30 minutes rest (pre) and at 0, 30, 60, and 120 minutes after a 30-minute walking exercise. Serum COMP concentration was analyzed by commercial ELISA. Physical activity duration (hours) was measured using an activity monitor for 7 consecutive days, and physical activity level (metabolic equivalent of the task (MET)) was quantified using the International Physical Activity Questionnaire (IPAQ). The reponse of COMP levels to the walking exercise was modelled using linear mixed-effects regression models. The association between physical activity and overall serum 